\title{
Erections on walking as a symptom of spinal canal stenosis
}

\author{
ANTHONY HOPKINS, * CHARLES CLARKE,* GILES BRINDLEY† \\ From the Department of Neurological Sciences St Bartholomew's Hospital, ${ }^{*}$ and the Institute of Psychiatry $\dagger$ \\ The Maudsley Hospital, London, UK
}

SUMMARY Two patients reported that on walking they developed tingling and weakness of the legs and penile erections. The symptoms proved to be due to stenosis of the lumbar canal, and were relieved by operative decompression.

Since the description by Verbiest in 1954 of spinal canal stenosis, ${ }^{12}$ and the recognition of reversible neurological deficits in the lower limbs on walking, ${ }^{3}$ the syndrome of "claudication of the cauda equina" has been increasingly recognised. ${ }^{4}$ We have encountered two patients with undoubted canal stenosis whose principal symptoms were unwanted erections on walking. Neurologists are more used to hearing of loss of erectile potency as a manifestation of intramedullary or extramedullary disease. We record these cases in the expectation that there may be other patients with an identical though unrecognised problem.

\section{Case reports}

\section{Case 1-H}

This married white security manager was seen at another hospital when aged 57 years. He was then complaining of pain in his left hip on walking, radiating to the left thigh and leg. He said that the legs tingled and became numb on walking, and that, if he continued, his testicles were similarly affected. A diagnosis of ischaemia of the cauda equina was suggested by the doctor whom he then saw. Plain films of the lumbar spine showed "degenerative changes". A spinal support was supplied, which gave him some relief, but no further action was undertaken. Over the next 2 years, the distance that he could walk without discomfort declined to about 50 metres. Five years after the onset of symptoms, at the age of 62 , he noted that after walking about 50 metres, his penis became erect. The patient was adamant that the

Address for reprint requests: Dr Anthony Hopkins, Department of Neurological Sciences, St Bartholomew's Hospital, London EC1A 7BE, UK.

Received 16 January 1987. Accepted 28 February 1987 erection was unwanted, and unaccompanied by any libidinous thoughts. If he sat down the leg pains were relieved, and the erection subsided over a few minutes. He had had no difficulties with sexual intercourse, and no urinary symptoms. He smoked 40 cigarettes a day.

Examination showed a generally fit man. All peripheral pulses were palpable, the pulse was regular, and the blood pressure $160 / 90 \mathrm{~mm} \mathrm{Hg}$. There were no arterial bruits. At rest there were no neurological signs but, after walking about 50 metres, $\mathrm{Mr} \mathrm{H}$ developed an erection and, when examined immediately afterwards, there was weakness of the extensors of both feet, and impairment of joint position sense at the left hallux. These signs persisted for only about 20 seconds after becoming supine, but were reproducible. The only other sign was a lumbar hernia, which was the reason for his inpatient admission prior to neurological consultation.

Radiculography showed a tight stenosis of the lumbar canal, with a moderate constriction at $\mathrm{L} 2 / 3$, severe constriction at $\mathrm{L} 3 / 4$, and a total block at $\mathrm{L} 4 / 5$ (fig 1). A dilated vein was seen within the cauda equina above the constriction. There was no significant change following exercise, but by then most of the contrast had disappeared.

Neurosurgical decompression of the lumbar spine was undertaken by Mr PJ Ward. The laminae of L3 and LA were seen to be thickened, and the texture of the bone was hard. Much of these laminae was removed. The ligamentum flavum was prominent. The spinal canal had a trefoil shape. There was no significant protrusion of intervertebral discs.

He was reviewed approximately 3 months later. He said that the result of the operation had been "fantastic". He could walk two or three miles without pain in the legs, and without any erection. Erections and intercourse continued normally. He had occasional low back pain, but no other symptoms. Eight months after operation the patient noted some tingling in the fingertips of the left hand. Electrodiagnostic studies showed absent sensory action potentials derived from both median and ulnar nerves in both upper limbs, with normal motor conduction, though a slightly pro- 


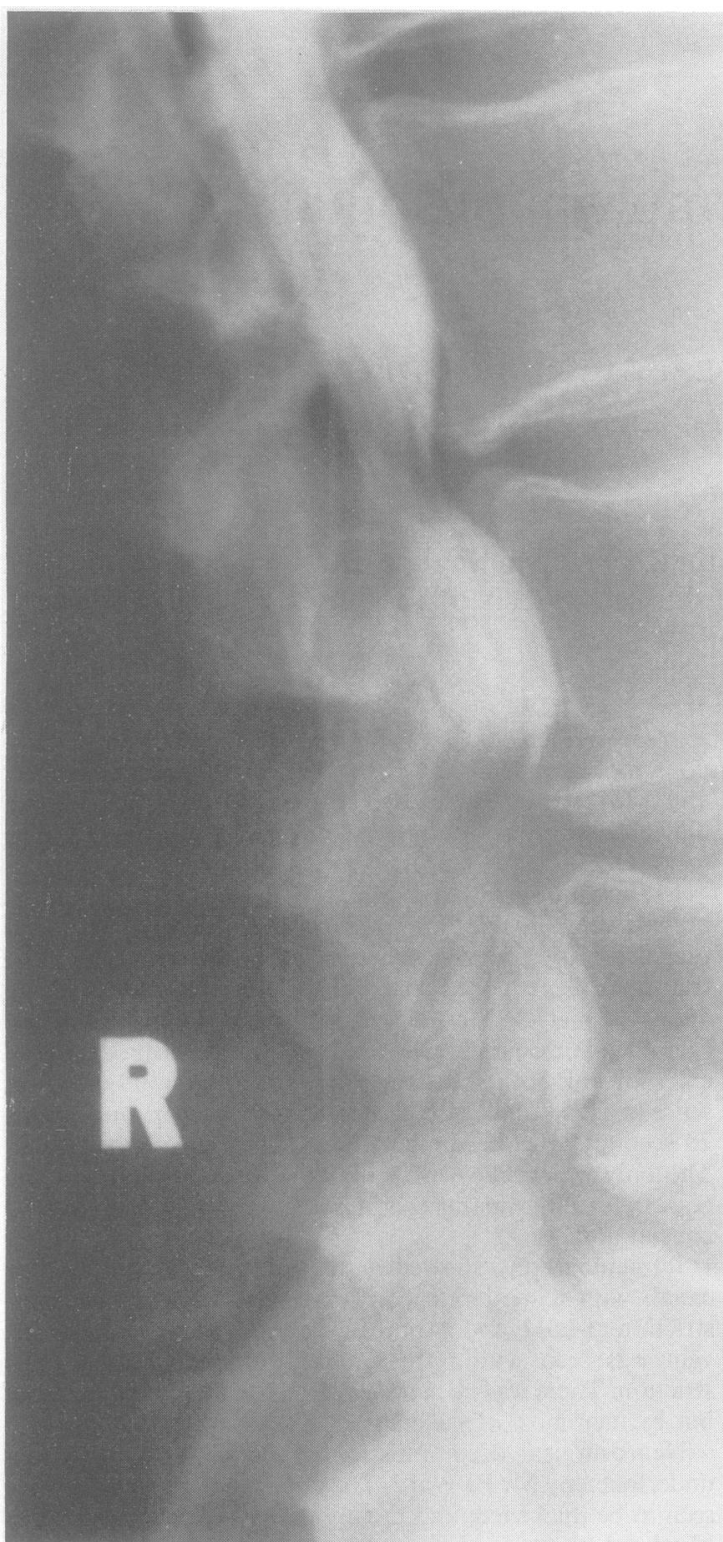

Fig 1 Preoperative radiculogram of Case 1. Note the "waisting" of the column of contrast.

longed distal latency in each median nerve $(5.6$ and $5.7 \mathrm{~ms})$. A normal blood sugar, serum B12, serum electrophoresis, thyroid function screen and a negative VDRL were the only investigations undertaken and, when seen 3 months later the symptoms were settling. It was presumed that he had had a mild peripheral neuropathy during this time.

Case 2-W

This 34 year old black carpenter noted cramp-like feelings in both legs on walking home from work. On each of many occasions over one year his legs felt heavy, numb and tingled, so that he was unable to control them. Numbness and tingling on exercise extended into his buttocks and genitalia. $\mathrm{He}$ developed an erection at the same time. Erections also developed when he had been kneeling at his work for half an hour or more. The erection, and the symptoms in the legs, subsided over 5 to 10 minutes as he rested.

There were no abnormal neurological signs on examination. Lumbar myelography was carried out with difficulty. The column of contrast was narrowed and compressed, with a particularly prominent anterior impression at the $\mathrm{L} 4 / 5$ level. There were prominent vessels above the compression.

Professor Valentine Logue undertook a laminectomy of L3-4-5. The L5 lamina was extremely thick, flattening and compressing the theca. There was similar but less prominent compression at L4. The canal was conspicuously narrow. In addition, a large soft bulging disc at L4/5 contributed substantially to the narrowing of the canal. A large amount of soft degenerate disc material was removed. Following operation all symptoms resolved. Erections and intercourse continued normally.

\section{Discussion}

Reversible neurological symptoms in the lower limbs on exercise have been known for many years. In 19110 Dejerine ${ }^{5}$ coined the term "claudication intermittente de la moelle épinière", a term taken up in the English $\overrightarrow{\overline{\mathrm{D}}}$ 을 language literature by Bergmark as "intermittent spinous claudication". 6 These patients developed upper motor neuron signs in the legs on exertion. At least some of the cases had a syphilitic spinal arteritis. In 1954 and 1955 Verbiest $^{12}$ described a number of patients who had radicular symptoms on exercise. $\mathrm{He}$ recognised that these were due to developmental "narrowness" of the lumbar vertebral canal. Blau and Logue $^{3}$ subsequently called the syndrome "intermittent claudication of the cauda equina". Other names include "neurogenic" 78 or "neurospinous" 4 claudication. The canal may be narrow by virtue of a congenitally small anterior-posterior diameter, or the particular placement and angle of the lateral masses, ${ }^{129}$ or by posterior protrusion of an intervertebral disc. ${ }^{3}$ The condition is more common in males, and in black people than white. Symptoms very often arise when a disc protrudes into a congenitally narrow canal, as in our Case 2. Occasionally, the canal is compromised by hypertrophy of bone posteriorly. ${ }^{10}$

Significant canal stenosis can sometimes be detected by plain radiological examination, ${ }^{8}$ but significant anterior-posterior stenosis can be overlooked. The inter-pedicular distance is usually normal, though at the lower part of the normal range. Radiculography, as in our cases, offers clear visualisation of the abnormality, but we have seen cases in 


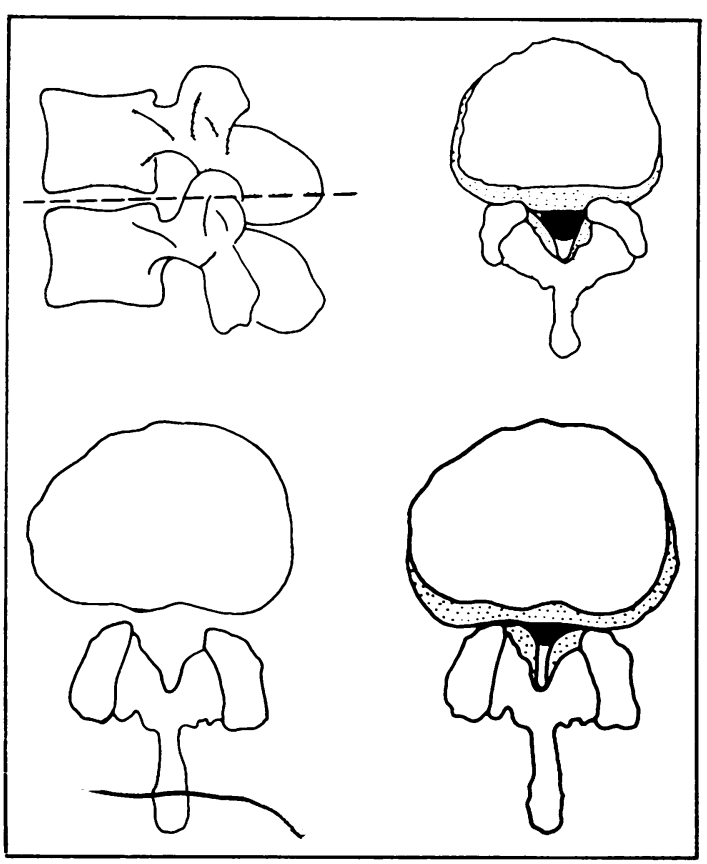

Fig 2 Top: On the left is emphasised the relationship between two adjacent vertebrae. The maximal stenosis is usually at the level indicated by the dotted line. On the right is seen a CT image corresponding to this level. The dotted areas represent the soft tissues, the disc, and the ligamentum flavum. The black area is dural sac, and the white area dorsal to the sac is fat. Bottom: Tracings of a CT scan from the L3-L4 level of a 69 year old man, showing the discrepancy between the size of the dural sac and the size of the bony canal. Reproduced by kind permission of DM Spengler, and the publishers of "Spine". 12

which lumbar radiculography has failed, presumably because the roots of the cauda equina are so tightly packed that no spinal fluid returns through the needle, and the operator does not proceed to inject contrast.

Computed tomographic analysis of the morphology of the lumbar spinal canal has been undertaken by Lancourt and colleagues, ${ }^{11}$ and by Schonstrom and colleagues. ${ }^{12}$ In fig 2 , reproduced from the paper by Schonstrom and colleagues, dotted areas represent soft tissues, the disc and ligamentum flavum, and the white area fat. Using measurements derived from such CT slices, Schonstrom and colleagues found that there was no correlation between the anterior-posterior diameter or cross-sectional area of the bony canal and the cross-sectional area of the dural sac. There was, however, good correlation between the anterior-posterior diameter of the dural sac and its cross-sectional area. In more than $80 \%$ of patients the maximal stenosis was at the level of the facetal joints (fig 2), but hypertrophic and degenerative changes in these joints are by no means the most frequent cause of canal stenosis, thickening of the ligamentum flavum and prolapsed intervertebral discs being more common in this series. ${ }^{12}$

The usual symptoms of neurospinous claudication are pain and tingling paraesthesiae in the distribution of the lower lumbar and sacral dermatomes on walking, relieved by rest. Weakness of muscles leading to foot-drop or a waddling gait ${ }^{3}$ may occur if the subject persists in walking after the onset of symptoms. The lower limb reflexes may be preserved, and may be lost on exercise. In some subjects pain may be such a predominant feature that there may be confusion with true arterial claudication. Clinical differences between vascular and neurospinous claudication were summarised by Hawkes and Roberts. ${ }^{8}$ Many patients with neurospinous claudication have a long history of back pain, ${ }^{4}$ and may be so used to it that it does not figure prominently in the more recent history on presentation to a neurologist.

The two patients here reported are unusual in developing erections on walking as a symptom of lumbar canal stenosis, and these unwanted erections were relieved by decompression. In neurological practice, most compressive lesions cause conduction block resulting in "negative" symptoms of weakness, impairment of sensation or loss of tendon reflexes. Such phenomena are common in claudication of the cauda equina. ${ }^{8} \mathrm{~A}$ further "negative" symptom (faecal incontinence ${ }^{13}$ ) of spinal stenosis has recently been described.

It is possible to explain theoretically involuntary erection on the basis of failure of conduction in nerve roots. There is an anti-erectile sympathetic pathway. Blockade of this by alpha adrenoreceptor blocking drugs, such as phenoxybenzamine, injected into the corpora cavernosa, causes erection. ${ }^{14}$ However, this pathway leaves the spinal cord at T11-12; in our patients there was no evidence of significant compression as high as this. Tingling (a "positive" symptom) depends upon the generation of nerve impulses in sensory fibres ${ }^{15}$ and not upon blockade. If impulses can be generated by compression of sensory fibres, presumably they can also in reflex-afferent and autonomic efferent fibres. It is therefore possible that, in our patients, impulses were generated in parasympathetic erectile fibres lying in the S2 and S3 roots, stimulation of which causes erections in man. ${ }^{16}$ Alternatively, impulses may have been generated in reflex-afferent fibres in S2-3 roots. Mechanical stimulation of the penis causes erections in some men in the complete absence of sexual thoughts, as, for example, being fully occupied in solving a mathematical problem. ${ }^{17}$ Reflex erections also occur readily in spinal men, as long as the conus medullaris is spared. Both 
patients experienced tingling in sacral dermatomes on walking, including the genitalia. This suggests that generation of impulses in somatosensory afferent and erectile reflex afferent fibres is the most likely explanation in these patients for erections on walking in stenosis of the lumbar canal.

We thank Dr P Gautier-Smith for permission to publish details of Case 2, a patient who was under his care, and Professor Dan M Spengler for permission to reproduce fig 2 . The friends of Michael Wheeler provided some support in the preparation of this manuscript.

\section{References}

1 Verbiest $\mathrm{H}$. A radicular syndrome from developmental narrowing of the lumbar vertebral canal. J Bone Joint Surg 1954;36B:230-7.

2 Verbiest $H$. Further experiences in the pathological influence of a developmental narrowness of the bony lumbar vertebral canal. J Bone Joint Surg 1955; 37B:576-83.

3 Blau JN, Logue V. Intermittent claudication of the cauda equina - an unusual syndrome resulting from central protrusion of a lumbar intervertebral disc. Lancet $1961 ; \mathbf{i}: 1086$.

4 Editorial. Neurospinous claudication. Lancet 1985;2: 704.

5 Dejerine J. Le claudication intermittente de la moelle épinière. Presse Med 1911;19:981-4.
6 Bergmark G. Intermittent spinal claudication. Acta Med Scand 1950;246(suppl):30-6.

7 Evans JG. Neurogenic intermittent claudication. $\mathrm{Br}$ Med $J$ 1964;2:985-7.

8 Hawkes CH, Roberts GM. Lumbar canal stenosis. $\mathrm{Br} J$ Hosp Med 1980;23:498-505.

9 Epstein JA, Epstein BS, Lavine L. Nerve root compression associated with narrowing of the spinal canal. J Neurol Neurosurg Psychiatry 1962;25:165-76.

10 Joffe R, Appleby A, Arjona V. "Intermittent ischaemia" of the cauda equina due to stenosis of the lumbar canal. J Neurol Neurosurg Psychiatry 1966;29:315-8.

11 Lancourt JE, Glen WV, Wiltse LL. Multiplanar computerised tomography in the normal spine, and in the diagnosis of spinal stenosis. Spine 1979;4:379-90.

12 Schonstrom NSR, Bolander N-F, Spengler DM. The pathomorphology of spinal stenosis as seen on CT scans of the lumbar spine. Spine 1985;10:806-11.

13 Foster OJF, Harrison MJG, Crockard HA. Exercise provoked faecal incontinence in spinal stenosis. $J \mathrm{Neu}$ rol Neurosurg Psychiatry 1987;50:362-3.

14 Brindley GS. Cavernosal alpha-blockade: a new technique for investigating and treating erectile impotence. Br J Psychiatry 1983;143:332-7.

15 Ochoa JL, Torebjörk HE. Parasthesial firm ectopic impulse generation in human sensory nerves. Brain 1980;103:835-53.

16 Brindley GS, Polkey CE, Rushton DN, Cardozo L Sacral anterior root stimulators for bladder control in $\bar{\omega}$ paraplegia: the first 50 cases. $J$ Neurol Neurosurgd Psychiatry 1986;49:1104-14.

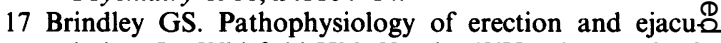
lation. In: Whitfield HN, Hendry WH, eds. Textbook of Genito-Urinary Surgery. Vol 2. Edinburgh Churchill-Livingstone, 1083-94. 